\title{
THE VALUE OF $H_{0}$ FROM THE TF DIAMETER RELATION
}

\author{
G. THEUREAU \\ ARPEGES/URA1757 \\ Paris-Meudon Observatory, 92195 Meudon cedex, France
}

Our KLUN sample (6600 spirals) is presently the largest homogeneous catalogue for using the Tully-Fisher (TF) relation as distance indicator, to study both the Hubble constant and peculiar velocities at the scale of the local Universe up to $z=0.025$.

Due to the internal dispersion of the distance criterion, and the flux limitation of every catalogue, the Malmquist bias leads to a progressive under-estimation of derived distances $d_{T F}$. Moreover, objects with different $\log V_{m}$, different Galactic extinction correction, different opacity correction, different morphological type or mean surface brightness consist in different classes with respect to the bias behaviour against true distance. The normalized distance $\left(d_{n}\right)$ method, by superimposing the different bias curves corresponding to theses different classes of objects in the same diagram $\log H=\log \left(V / d_{T F}\right)$ vs. $d_{n}$, allows us to visualize the unbiased part of the sample, which appears as a plateau at short $d_{n}$. Moreover, the use of morphological types or mean surface brightnesses as additional parameters has reduced the TF scatter by $30 \%$, and then extended the unbiased range up to farther distances. The zero point variations of the $\mathrm{TF}$ regression with type or brightness is physically explained by the variation of the mass ratios of the different components constituting a spiral galaxy (Theureau et al. 1997, A\&A 319, 435; Theureau 1997, A\&A in press).

The slope of the TF relation is estimated through an iterative way from the data of the unbiased domain. The absolute zero point is fixed using a set of cepheid distances collected from literature (mainly from the HST project). From the unbiased domain of our field galaxies sample, we derived $H_{0}=55 \pm 5$. The new absolute calibration of the Period-Luminosity relation provided by HIPPARCOS parallaxes (Paturel et al. 1997, MNRAS in prep) leads to a $10 \%$ smaller value of $H_{0}=49 \pm 6$. This is a global value in perfect agreement with the result derived from SNIa standard candles which reach $z=0.1$ (Theureau et al. 1997, A\&A 322, 730). 